\title{
Sham procedures and the ethics of clinical trials
}

\author{
Franklin G Miller PhD $^{1}$ Ted J Kaptchuk OMD ${ }^{2}$
}

J R Soc Med 2004;97:576-578

Placebo-controlled trials of pharmacological treatments are typically conducted double-blind - that is, neither the patients nor the investigators know whether the substance administered is the agent under investigation or placebo. The process of masking treatment assignment is generally considered ethically acceptable provided that the 'shared ignorance' ${ }^{1}$ has been made clear in the consent process. However, in circumstances where a surgical or medical procedure itself constitutes the treatment, a randomized placebo-controlled trial raises different issues. Here only the patient-subject is kept in ignorance, and the clinician, who can distinguish active from inactive treatment, may be required to engage in active deception. Examples are surgical operations, acupuncture and specific methods of psychotherapy. Recent papers on the ethics of such trials have focused on the risk-benefit assessment of invasive sham interventions. ${ }^{2-11}$ Little attention has been given to the psychological and ethical concerns generated by the need for deliberate deception.

\section{EXAMPLES OF SHAM PROCEDURES}

The following three examples illustrate the use of active deception in sham procedure trials. In a study of transmyocardial laser treatment administered during cardiac catheterization, laser treatment was compared with no treatment. ${ }^{12}$ All patients were blindfolded and sedated. In the case of those in the placebo arm, the laser equipment was wheeled into the hospital room and laser treatment was discussed as it would have been with the real treatment, but no treatment was administered. Similarly, in a placebocontrolled trial of arthroscopic surgery for osteoarthritis of the knee, those in the placebo arm received only a small skin incision under sedation without the arthroscopic intervention. ${ }^{13}$ For the sham procedure the surgeoninvestigator asked to be given the usual instruments and manipulated the knees of these individuals as if the real treatment was being administered; in addition, water was splashed to mimic the sounds of lavage. Thirdly, in sham-

${ }^{1}$ Department of Clinical Bioethics, Clinical Center, National Institutes of Health Building 10, Room 1C118, Bethesda, MD 20892-1156; ${ }^{2}$ Osher institute, Harvard Medical School, Boston, MA, USA

Correspondence to: Franklin G Miller PhD

E-mail: fmiller@nih.gov controlled trials of acupuncture, clinician-investigators commonly use equipment designed to look and feel exactly like standard treatment except that the needles do not penetrate the skin. The device is a miniature 'magic sword' whereby the needle retracts up the needle shaft. ${ }^{14}$ Despite being ethically grounded by a sound methodological rationale, despite review and approval by a research ethics committee, and despite the informed consent of research subjects, sham interventions are apt to cause moral discomfort in clinician-investigators. Trained to perform invasive interventions only for the medical benefit of patients, they find themselves administering fake procedures. Moreover, they must manipulate the performance so as to create a false belief in patientsubjects that a real procedure is being administered - a deception that may have to be maintained in follow-up visits.

\section{PERSONAL EXPERIENCE IN ACUPUNCTURE TRIALS}

One of the authors (TK) has often used sham acupuncture devices in randomized controlled trials. Yet, despite being a full-time researcher, he braces himself before opening the sealed opaque randomization envelope for every patient. $\mathrm{He}$ must remember not to change his demeanour if the patient, with whom he has already developed a clinical relationship, is randomized to sham treatment. He has to demonstrate identical concern, compassion, kindness, and attention whether the patient is receiving genuine or placebo treatment. Such moral concerns are forgotten as the intervention takes on its own momentum: awareness that a good research-actor is needed to produce valid data becomes an important reinforcement of the temporary amnesia. Yet on each subsequent visit by the same patient the researcher is likely to experience a mini version of this cycle of uneasiness and forgetfulness. He must remind himself repeatedly of the scientific reasons why 'the show must go on'.

\section{ETHICAL REFLECTIONS}

Some might argue that sham procedure trials involving active deception are inherently unethical - that, however important the matter to be investigated, clinician- 
investigators should not engage in fraud. But is the conduct described above fraudulent in a way that is ethically objectionable? It would be truly fraudulent for a clinician to perform a fake therapy in the guise of competent medical care. In contrast, sham procedure trials are scientific experiments in which the active deception is methodologically necessary to produce valid results. Understood as research interventions that carry risks to individuals without a prospect of compensating benefit to them, sham procedures are no different in principle from common research interventions for determining outcomes such as blood-taking, lumbar puncture, or biopsy. ${ }^{11}$ Most importantly, the use of sham interventions does not violate the rights of patient-subjects provided that they have been adequately informed that they will receive either a real or a sham intervention and that efforts will be made to make the sham procedure indistinguishable from the real treatment under investigation. The authorization beforehand by research subjects makes the difference between legitimate and unethical deception. ${ }^{15}$

So why the moral discomfort experienced by clinicianinvestigators? Such reactions illuminate the ethically significant differences between clinical trials and medical care. Any randomized controlled trial can be regarded as in breach of clinical ethics in that the aim is to answer questions about groups of patients rather than provide personalized care: ${ }^{16,17}$ the concealment of treatment allocation is justified by the potential value of the knowledge to be gained rather than the medical interests of these patients. For trials involving sham procedures, clinician-investigators face this ethical conflict in its starkest form; those who conduct trials of pharmaceutical agents have less difficulty in conflating their scientific and clinical roles.

Professional integrity in clinical research calls for an appreciation of how scientific experimentation involving patient-subjects necessarily departs from the ethics of medical care, without thereby necessarily becoming unethical. ${ }^{18}$ Professional integrity for investigators conducting sham-procedure trials is preserved only by recognition that they are operating primarily as scientists, not as clinicians. Undivided loyalty to patient care is the fundamental credo of medical ethics. Although clinicianinvestigators often wear white coats, they cannot honestly subscribe to this notion. The ethics of clinical research calls for a complex balancing of commitments to rigorous science, improvement of medical care and protection of research subjects from undue risks of harm and exploitation. ${ }^{19}$ Moral perspectives drawn from the ethics of medical care, which make sham procedure trials appear ethically suspect, distort the ethics of clinical research. Unfortunately, these ethical distinctions between medical care and clinical research are often blurred or confused. For example, the Declaration of Helsinki, in its 32 ethical principles for medical research, specifies that 'The health of my patient will be my first consideration'. ${ }^{20}$ This is not appropriate ethical guidance for physicians conducting clinical research. Just as participants in clinical trials are neither patients receiving personalized medical therapy nor human guinea-pigs, so clinical investigators in the conduct of research are neither clinicians nor laboratory scientists. In clinical practice a sham medical procedure would be fraudulent and deplorable; in research such activities can be legitimate, and this outlying research practice underscores the important ethical differences between clinical trials and medical care.

Acknowledgment T J Kaptchuk was partially supported by grants Nos. 1R01 AT01414-01 and 1R01 AT00402-01 from the National Center of Complementary and Alternative Medicine at the National Institutes of Health.

Note: The opinions expressed are those of the authors and do not reflect the position or policy of the National Institutes of Health, the Public Health Service or the Department of Health and Human Services.

\section{REFERENCES}

1 Kaptchuk TJ. Intentional ignorance: a history of blind assessment and placebo controls in medicine. Bull Hist Med 1998;72:389-433

2 Freeman TB, Vawter DE, Leaverton PE. Use of placebo surgery in controlled trials of a cellular-based therapy for Parkinson's disease. $N$ Engl J Med 1999;341:988-91

3 Macklin R. The ethical problem with sham surgery in clinical research. N Engl J Med 1999;341:992-6

4 Dekkers W, Boer GB. Sham neurosurgery in patients with Parkinson's disease: is it morally acceptable? J Med Ethics 2001;27:151-6

5 Gillett GR. Unnecessary holes in the head. IRB 2001;23:1-6

6 Albin RL. Sham surgery controls: intracerebral grafting of fetal tissue for Parkinson's disease and proposed criteria for use of sham surgery controls. J Med Ethics 2002;28:322-5

7 Clark PA. Placebo surgery for Parkinson's disease: do the benefits outweigh the risks? J Law Med Ethics 2002;30:58-68

8 Horng S, Miller FG. Is placebo surgery ethical? $N$ Engl $J$ Med 2002;347:137-9

9 London AJ, Kadane JB. Placebos that harm: sham surgery controls in clinical trials. Stat Methods Med Res 2002;11:413-27

10 Weijer C. I need a placebo like I need a hole in the head. J Law Med Ethics 2002;30:69-72

11 Miller FG. Sham surgery: an ethical analysis. Am J Bioethics 2003;3: $41-8$

12 Winslow R. Placebo study questions effectiveness of laser heart treatment. Wall Street J, 19 October 2000

13 Moseley JB, O'Malley K, Petersen NJ, et al. A controlled trial of arthroscopic surgery for osteoarthritis of the knee. N Engl $\mathrm{J}$ Med 2002;347:81-8

14 Streitberger K, Kleinhenz J. Introducing a placebo into acupuncture research. Lancet 1998;352:364-5 
J O U R N A L O F T H E R O Y A L S O C I ETY O F M E I CINE Volume 97 December 2004

15 Wendler D, Miller FG. Deception in the pursuit of science. Arch Intern Med 2004;164:597-600

16 Miller FG, Rosenstein DL. The therapeutic orientation to clinical trials. N Engl J Med 2003;348:1383-6

17 Miller FG, Brody H. A critique of clinical equipoise: therapeutic misconception in the ethics of clinical trials. Hastings Center Rep 2003;33:19-28
18 Miller FG, Rosenstein DL, DeRenzo EG. Professional integrity in clinical research. JAMA 1998;280:1449-54

19 Emanuel EJ, Wendler D, Grady C. What makes research ethical? JAMA 2000;283:2701-11

20 World Medical Association. Declaration of Helsinki: Ethical Principles for Medical Research Involving Human Subjects. JAMA 2000;284:3043-5 